\title{
The Nature and Realization of Quantum Entanglement
}

\author{
Bin Liang ${ }^{1}$ \\ ${ }^{1}$ College of Science, Chongqing University of Posts and Telecommunication, Chongqing 400065, China \\ Correspondence: Bin Liang, College of Science, Chongqing University of Posts and Telecommunication, \\ Chongqing 400065, China. E-mail: lianji@ohsu.edu
}

Received: October 9, 2016

Accepted: October 25, 2016

Online Published: November 30, 2016

doi:10.5539/apr.v8n6p96

URL: http://dx.doi.org/10.5539/apr.v8n6p96

\begin{abstract}
This paper analyses the nature of quantum entanglement, proves the quantum entanglement is not action at a distance, proposes a scheme to realize quantum entanglement, explains that the quantum entanglement is not action at a distance and the non-cloning theorem of quantum state ensure the quantum mechanics is consistent with relativity and make the superluminal communication could not happened.
\end{abstract}

Keywords: Quantum Entanglement, teleportation, Quantum Communication

\section{Introduction}

Is the quantum entanglement a spooky action at a distance? This problem has caused heated debate for a long time (Schrödinger, 1935; Einstein, Podolsky, \& Rosen, 1935; Li, 2000). It is not a simple physics problems, also involves philosophy.

The quantum entanglement is the core concept of quantum information. No real quantum entanglement, no real quantum communication.

The concept of quantum entanglement was put forward by Schrodinger in 1935, and is called the essence of quantum mechanics (Schrödinger, 1935). In the same year, Einstein, Podolsky and Rosen (EPR) suggested that quantum mechanics might be incomplete on the basis of that (Einstein, Podolsky, \& Rosen, 1935), and so caused the in-depth study of people to quantum entanglement and the essence of quantum mechanics. However, since then, the experimental results do not support the EPR's viewpoint (Li, 2000; Zhang, 2006).

It must be noted that Hidden parameter theory represented by Bell inequality (Bell, 1964) is essentially deterministic, that is not accord with the experiments proves only again that the movement of micro particles is not deterministic rather than quantum entanglement is an action at a distance.

According to the Schmidt decomposition theorem (Li, 2000; Nielsen \& Chuang, 2004; Hughes \& Heinrichs, 2004; Zhang, 2006) of quantum mechanics, the pure state $|\psi\rangle$ of a composite system composed of two arbitrary subsystems can be expand as

$$
|\psi\rangle=\sum_{m} \sqrt{\rho_{m}}\left|\phi_{m}^{(1)}\right\rangle\left|\phi_{m}^{(2)}\right\rangle,
$$

where $\left|\phi_{m}^{(1)}\right\rangle$ and $\left|\phi_{m}^{(2)}\right\rangle$ is a pair of eigenstates of density operator $\hat{\rho}^{(1)}$ and $\hat{\rho}^{(2)}$ with the common eigenvalue $\rho_{m}$ and called a pair of dual states, while the number of terms of the expansion (1) is called the Schmidt number, and there is

$$
\sum_{m} \rho_{m}=1
$$

The Schmidt expansion to satisfy the equation above is called complete, as shown in equation (1), and we discuss only the complete Schmidt expansion in this article.

According to the literatures of quantum information, if the Schmidt number is greater than 1, the measurement to a subsystem of the composite system makes the put state $|\psi\rangle$ become instantaneously into some term in the expansion with different probability $\rho_{m}$, and this is called the quantum entanglement. In other words, the measurement to a subsystem changes instantaneously the state of another subsystem. So, the quantum entanglement seems to be superluminal. But, the interaction between materials is not superluminal according to relativity. The contradiction is sharp, how to solve? 
In the following we firstly analyze the nature of quantum entanglement, prove the quantum entanglement is not action at a distance and propose a scheme to realize quantum entanglement, and then explain that the quantum entanglement is not action at a distance and the non-cloning theorem of quantum state ensure together the quantum mechanics is consistent with relativity and make the superluminal communication could not happened.

\section{The Nature of Quantum Entanglement}

(1) The quantum entanglement is not action at a distance.

For the famous Schrodinger cat, the Schmidt decomposition of the pure state $|\psi\rangle$ of the composite system composed of a cat and a poison bottle is

$$
|\psi\rangle=\frac{1}{\sqrt{2}}[|b c\rangle|c l\rangle+|b o\rangle|c d\rangle],
$$

where $|b c\rangle,|b o\rangle,|c l\rangle$ and $|c d\rangle$ is the state of the closed poison bottle, open poison bottle, live and dead cat, respectively. The reason of cat dead is that it contacted the poison gas from the bottle when the bottle was open. If there is not the poison gas, the state of cat does not change no matter the bottle was open or not. Namely, the entanglement of equation (3) is only an artificial mathematical combination without real significance if there is not the poison gas.

It is a pair of entangled photons prepared with SPDC of BBO crystal in Figure 1 which is often quoted in the literatures of quantum information (Nielsen \& Chuang, 2004; Hughes \& Heinrichs, 2004). According to these literatures, the two photons flying respectively in the top and bottom cone are in the entangled state due to the photon indiscernibility within the overlapping area of the two cones, their polarization entanglement state is

$$
|\psi\rangle_{ \pm}=\frac{1}{\sqrt{2}}\left[\left|H^{(1)}\right\rangle\left|V^{(2)}\right\rangle \pm\left|V^{(1)}\right\rangle\left|H^{(2)}\right\rangle\right]
$$

where $|H\rangle,|V\rangle$ are the horizontal and vertical polarization state of the photons, respectively. The equation above shows that as long as the polarization state of one photon has been detected, the polarization state of another photon would know immediately when two photons are separated far. But there is an implicit assumption: the polarization state of each photon is changeless in motion, and this implies there is not interaction between the two photons, they are not really entangled. In fact, the entanglement as shown in the Figure 1 is only the synonym of indiscernibility of photons, and such photons of changeless state can't transfer any information.

In a word, the quantum entanglement is real only when there is real interaction between the subsystems. Since all of the four interactions between materials are not action at a distance, so the quantum entanglement is not action at a distance.

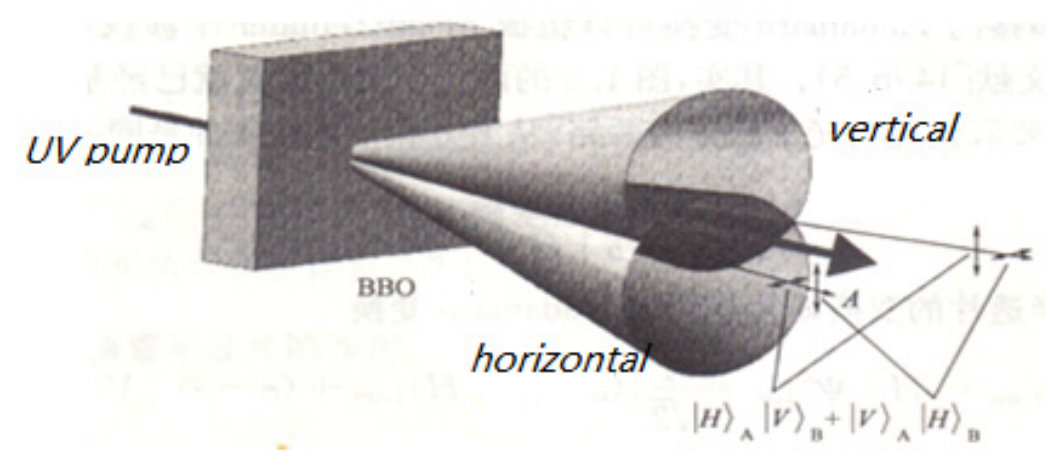

Figure 1. The pair of entangled photons prepared with SPDC of BBO crystal

(2) The entanglement does not change the probability to find each qubit state.

Assume the coherent state of qubit A and B is

$$
\begin{aligned}
& |A\rangle=\alpha|0\rangle+\beta|1\rangle, \\
& |B\rangle=\delta|2\rangle+\gamma|3\rangle,
\end{aligned}
$$


respectively, where the four quantum states $|0\rangle$ and $|1\rangle,|2\rangle$ and $|3\rangle$ are orthogonal and normalization, $\alpha^{2}+\beta^{2}=1, \delta^{2}+\gamma^{2}=1$. The entangled state of the two qubits is

$$
|A B\rangle=|A\rangle|B\rangle=\alpha \delta|02\rangle+\alpha \gamma|03\rangle+\beta \delta|12\rangle+\beta \gamma|13\rangle .
$$

The probability to find qubit state $|0\rangle$ in the entangled state is

$$
\left.|\langle 0 \mid A B\rangle|^{2}=|\alpha \delta| 2\right\rangle+\left.\alpha \gamma|3\rangle\right|^{2}=\alpha^{2}\left(\delta^{2}+\gamma^{2}\right)=\alpha^{2},
$$

and this is identical with the probability to find qubit state $|0\rangle$ in the coherent state $|A\rangle$ before the entangled. Similarly, the probability to find other qubit state in the entangled state of equation (6) is identical with that before the entangled. Therefore, the entanglement does not change the probability to find each qubit state.

\section{The Realization of Quantum Entanglement}

As said previously, the entanglement is real only when there is real interaction between the subsystems. The following is a scheme to realize quantum entanglement.

It is well known that the particle radiated laser pulse transited from high to low energy level, and the particle absorbed laser pulse transited from low to high energy level. Thereby the laser pulse could be for the use of making particles in entanglement state. In fact, the laser pulse had been considered for the use of change the qubit state in the realization scheme of quantum computer, for example, ion trap scheme, QED scheme and quantum dots scheme (Hughes \& Heinrichs, 2004; De Caro \& Garuccio, 1994; Peres, 1996; Kwiat et al., 1995).

Image the ground and excited state of two identical particles located in two different places is $|0\rangle_{i}$ and $|1\rangle_{i}$ $(i=1,2)$, respectively, and the entangled state is

$$
|\psi\rangle=\frac{1}{\sqrt{2}}\left[|1\rangle_{1}|0\rangle_{2}+|0\rangle_{1}|1\rangle_{2}\right]
$$

the interaction Hamiltonian is $(\hbar=1)$

$$
\hat{H}_{i}=\omega_{0} e^{i \phi} a_{1} a_{2}^{+},
$$

where $\omega_{0}=E_{1}-E_{0}$ is the difference of two energy levels of the particle, namely the energy of the pulse photon. If the energy levels are that of electronic spin, then there is $\omega_{0}=E_{1}-E_{0}=\frac{e}{m} H(\mathrm{SI}), H$ the magnetic intensity; and $a_{i}, a_{i}^{+}$is the annihilation and creation operator of photon, and $\phi$ is the phase of the laser. So there is

$$
\hat{H}_{i}|\psi\rangle=\frac{\omega_{0}}{\sqrt{2}} e^{i \phi}|0\rangle_{1}|1\rangle_{2} .
$$

Obviously, the Hamiltonian of equation (9) describes that the particle 1 releases a photon and transits from the excited state to the ground state, while the particle 2 absorbs that photon and transits from the ground state to the excited state. This is the single-direction entanglement. And the Hamiltonian of double-direction entanglement

$$
\hat{H}_{i d}=\omega_{0}\left(e^{i \phi} a_{1} a_{2}^{+}+e^{-i \phi} a_{1}^{+} a_{2}\right),
$$

where the first term is just the equation (9), and the second term describes that the particle 2 releases a photon and transits from the excited state to the ground state, while the particle 1 absorbs that photon and transits from the ground state to the excited state, thus the performance of double-direction entanglement to the entangled state $|\psi\rangle$ gives

$$
\hat{H}_{i d}|\psi\rangle=\frac{\omega_{0}}{\sqrt{2}}\left(e^{i \phi}|0\rangle_{1}|1\rangle_{2}+e^{-i \phi}|1\rangle_{1}|0\rangle_{2}\right) .
$$

This shows that the result of double-direction entanglement is that the system has returned to its initial state, only the relative phase of the two terms changes by $2 \phi$. Obviously, both the equation (8) and (12) is the greatest entangled state, and the probability to find each qubit state does not change due to the entanglement. 


\section{Quantum Entanglement and Quantum Communication}

We know that the key of realization of quantum communication is the realization of quantum teleportation, and the key of realization of quantum teleportation is the realization of quantum entanglement. The explanation is as follows (Li, 2000; Nielsen \& Chuang, 2004; Hughes \& Heinrichs, 2004; Zhang, 2006).

Assume each of Alice and Bob has one qubit of the greatest entangled state $\left|\phi^{+}\right\rangle=\frac{1}{\sqrt{2}}(|00\rangle+|11\rangle)$,

and Alice has the first qubit. The unknown qubit state of Alice wants to transmit to Bob is

$$
|\alpha\rangle=a|0\rangle+b|1\rangle
$$

where both $a, b$ is the unknown coefficient. Thus, the initial state of the system consisted of the three qubits is

$$
\left|\Psi_{0}\right\rangle=|\alpha\rangle\left|\phi^{+}\right\rangle=\frac{1}{\sqrt{2}}(a|000\rangle+b|100\rangle+a|011\rangle+b|111\rangle) .
$$

Since there exists quantum entanglement, Alice performs the CNOT operation to the first two qubits of the above equation, namely $|\alpha\rangle$ and her own qubits, and it gives

$$
\left|\Psi_{1}\right\rangle=\frac{1}{\sqrt{2}}(a|000\rangle+b|110\rangle+a|011\rangle+b|101\rangle) .
$$

And then the $\mathrm{H}$ door operation to the first qubit of the equation above gives

$$
\begin{aligned}
\left|\Psi_{2}\right\rangle & =\frac{1}{2}[a(|0\rangle+|1\rangle)|00\rangle+b(|0\rangle-|1\rangle)|10\rangle+a(|0\rangle+|1\rangle)|11\rangle+b(|0\rangle-|1\rangle)|01\rangle] \\
& =\frac{1}{2}[a|000\rangle+a|100\rangle+b|010\rangle-b|110\rangle+a|011\rangle+a|111\rangle+b|001\rangle-b|101\rangle] \\
& =\frac{1}{2}[|00\rangle(a|0\rangle+b|1\rangle)+|10\rangle(a|0\rangle-b|1\rangle)+|01\rangle(a|1\rangle+b|0\rangle)+|11\rangle(a|1\rangle-b|0\rangle)],
\end{aligned}
$$

where the following formula is used:

$$
\begin{aligned}
& H|0\rangle=\frac{1}{\sqrt{2}}(|0\rangle+|1\rangle), \\
& H|1\rangle=\frac{1}{\sqrt{2}}(|0\rangle-|1\rangle) 。
\end{aligned}
$$

Now Alice measures each of the first two qubits before one of the four superposition states of the equation (16) once, and $\left|\Psi_{2}\right\rangle$ becomes one of the four states with equal probability due to the existence of quantum entanglement. But the four states also could be obtained by the operations to the state $|\alpha\rangle$ as follows:

$$
\begin{aligned}
& I|\alpha\rangle=a|0\rangle+b|1\rangle, \\
& Z|\alpha\rangle=a|0\rangle-b|1\rangle, \\
& X|\alpha\rangle=a|1\rangle+b|0\rangle, \\
& -Y|\alpha\rangle=a|1\rangle-b|0\rangle .
\end{aligned}
$$

Therefore, the information of the first two qubit states of each term of equation (15) is correspondent with the above each operation one to one. Namely the above local operation of Alice has projected the Bob's qubit to one of the above four states due to the existence of quantum entanglement. And then Alice sends the obtained information about the first two qubit states to Bob through classical communication, and Bob has known that he should do the inverse transformation of which equation of the equation (18) according to the information from Alice and obtained the state $|\alpha\rangle$. In the transfer process above, the state $|\alpha\rangle$, namely the coefficient $a$ and $b$ are unknown always for Alice, so the transfer process is called the quantum teleportation. Obviously, the realization of the series of operations above is impossible without the real quantum entanglement, therefore, the key of realization of quantum teleportation is the realization of quantum entanglement. 


\section{Conclusion: Superluminal Communication is Impossible}

The above analyses the nature of quantum entanglement, proves the quantum entanglement is not action at a distance, proposes a scheme to realize quantum entanglement, and shows the key of realization of quantum teleportation is the realization of quantum entanglement. Also it must be noted that the quantum entanglement is not action at a distance and the non-cloning theorem (Wooters \& Zurek, 1982; Nielson \& Chuang, 2000; Pan, Chen, Żukowski, Weinfurter, \& Zeilinger, 2008; Xiong, Yu, Zhan, \& Zhang, 2016) of quantum state ensure together the quantum mechanics is consistent with relativity, and make the superluminal communication could not happened. The explanation is as follows.

If the quantum entanglement is a spooky action at a distance, when Alice wants to send the one bit of information to Bob, she could make instantaneously the qubits of Bob in the corresponding quantum state as long as she performs the proper operation to her own qubits. If Bob could clone reliably these unknown quantum states from Alice, he could know the information from Alice by copy and measurement for many times, and thus the superluminal communication is realized. But, since the quantum entanglement is not action at a distance and the unknown quantum state could be not reliable cloned, the quantum mechanics is consistent with relativity, the superluminal communication is impossible.

\section{References}

Bell, J. S. (1964). Physics Long Island City. NY, 1(195), 1.

De Caro, L., \& Garuccio, A. (1994). Reliability of Bell-inequality measurements using polarization correlations in parametric-down-conversion photon sources. Physical Review A, 50(4), R2803.

Einstein, A., Podolsky, B., \& Rosen, N. (1935). Can quantum-mechanical description of physical reality be considered complete?. Physical review, 47(10), 777.

Hughes, R., \& Heinrichs, T. (2004). A quantum information science and technology roadmap.

Kwiat, P. G., Mattle, K., Weinfurter, H., Zeilinger, A., Sergienko, A. V., \& Shih, Y. (1995). New high-intensity source of polarization-entangled photon pairs. Physical Review Letters, 75(24), 4337.

Li, C. Z. (2000). Quantum communication and quantum computation. National university of defense technology press.

Nielsen, M. A., \& Chuang, I. L. (2004). Quantum Computation and Quantum Information (Cambridge Series on Information and the Natural Sciences).

Nielson, M. A., \& Chuang, I. L. (2000). Quantum computation and quantum information. Cambridge University Press.

Pan, J. W., Chen, Z. B., Żukowski, M., Weinfurter, H., \& Zeilinger, A. (2008). Multi-photon entanglement and interferometry. arXiv preprint arXiv:0805.2853.

Peres, A. (1996). Separability criterion for density matrices. Physical Review Letters, 77(8), 1413.

Schrödinger, E. (1935). Die gegenwärtige Situation in der Quantenmechanik. Naturwissenschaften, 23(49), 823-828.

Wooters, W. K., \& Zurek, W. K. (1982). Quantum no-cloning theorem. Nature, 299, 802.

Xiong, P. Y., Yu, X. T., Zhan, H. T., \& Zhang, Z. C. (2016). Multiple teleportation via partially entangled GHZ state. Frontiers of Physics, 11(4), 1-8.

Zhang, Y. D. (2006). The physical principle of quantum information. Science press, China.

\section{Copyrights}

Copyright for this article is retained by the author(s), with first publication rights granted to the journal.

This is an open-access article distributed under the terms and conditions of the Creative Commons Attribution license (http://creativecommons.org/licenses/by/4.0/). 